\title{
BILIARY DISEASE IN PREGNANCY: A DIAGNOSTIC DILEMMA
}

\section{PRIYANKA H KRISHNASWAMY ${ }^{*}$, CAROL LORDEN ${ }^{2}$, SAMEH MAHMOUD $^{3}$, HARRY KALTSIDIS ${ }^{4}$, ROHIT ARORA ${ }^{5}$}

${ }^{1}$ Subspecialty Registrar in Urogynaecology, Queen Elizabeth University Hospital, Glasgow, Scotland. ${ }^{2}$ Internal Medicine Trainee, Wythenshawe Hospital, Manchester University NHS Foundation Trust, Manchester, United Kingdom. ${ }^{3}$ Speciality Registrar in Obstetrics and Gynaecology, Wythenshawe Hospital, Manchester University NHS Foundation Trust, Manchester, United Kingdom. ${ }^{4}$ Gastroenterology and Pancreatobiliary Medicine, Wythenshawe Hospital, Manchester University NHS Foundation Trust, Manchester, United Kingdom. ${ }^{5}$ Obstetrician and Gynaecologist and Honorary, Senior Clinical Lecturer, Wythenshawe Hospital, Manchester University NHS Foundation Trust, Manchester, United Kingdom. Email: priyanextdoor@yahoo.com

Received: 09 February 2021, Revised and Accepted: 20 March 2021

\section{ABSTRACT}

This is a case report of a 37-year-old female who presented with deranged liver function tests, epigastric pain, fevers, vomiting, mild hypertension, and proteinuria in pregnancy. This was on a background of gestational diabetes, dyspepsia, and previous cholecystectomy. This case report highlights the importance of considering various causes of liver pathology during pregnancy besides those specifically associated with pregnancy, as well as the necessity of early specialist input to investigate symptomatic choledocholithiasis in pregnancy.

Keywords: Biliary disease, Pregnancy, Choledocholithiasis, Diagnosis.

(C) 2021 The Authors. Published by Innovare Academic Sciences Pvt Ltd. This is an open access article under the CC BY license (http://creativecommons, org/licenses/by/4.0/) DOI: http://dx.doi.org/10.22159/ijms.2021v9i3.41050. Journal homepage: https://innovareacademics.in/journals/index.php/ijms

\section{INTRODUCTION}

Gallstone-related complications are relatively common during pregnancy, with reports of biliary sludge in $5-31 \%$ and cholelithiasis in $2-11 \%$ of expectant mothers [1]. About $1 \%$ of these women will develop complications secondary to cholelithiasis, which may lead to increased fetal and maternal morbidity and mortality (Ko et al., 2005) [2]. This case highlights the importance of considering a broad spectrum of hepatobiliary disorders during pregnancy, including choledocholithiasis and considering urgent endoscopic retrograde cholangiopancreatography (ERCP) as a safe option for those women with symptomatic complicated cholechodolithiasis.

\section{CASE REPORT}

A 35-year-old female, Gravida 5, Para 4, presented to antenatal clinic at 31 weeks of gestation with 3 days of epigastric pain and vomiting. She had a body mass index of 47 and a background of depression, migraine, dyspepsia, and previous cholecystectomy. She developed gestational diabetes during her pregnancy and received growth scans which were all normal. As she was high risk for thromboembolic disease in pregnancy, she was on prophylactic enoxaparin.

Initial observations revealed borderline hypertension and urine dipstick showed ketones $(4+)$ and proteinuria, but no glycosuria. She was admitted pending further tests for blood pressure monitoring, IV hydration, and antiemetics. On examination, she complained of epigastric pain, without any positive abdominal findings.

Liver function tests showed an alanine transferase (ALT) of $501 \mathrm{U} / \mathrm{L}$, with the rest of the parameters being normal along with normal renal function, coagulation, amylase, autoimmune antibody, and viral screen. Arterial blood gases showed a metabolic alkalosis consistent with protracted vomiting and hypovolemia. Cardiotocogram was normal and an abdominal ultrasound scan did not reveal a specific cause for the high ALT levels.

The raised ALT in association with epigastric pain, proteinuria, and hypertension raised the possibility of pre-eclampsia complicated by hemolysis, elevated liver enzymes, and low platelets syndrome. The lack of associated symptoms such as upper abdominal pain, decrease in urine output, persistent headache or blurring of vision, and tendency to bleed as well as relatively normal bloods made this less likely. Hepatobiliary causes of this presentation were also considered, and specialist input was sought. Amongst these differential diagnosis was acute fatty liver disease of pregnancy (AFLP), which is a relatively uncommon but lifethreatening cause of acute liver failure in pregnancy with mortality rates for fetus and mother ranging between 1-20\% [3]. However, the absence of hypoglycemia or a fulminant course of acute liver failure effectively excluded this important differential diagnosis. Obstetric cholestasis was felt to be unlikely given the LFT pattern, absence of pruritus, and presence of epigastric pain. The patient's symptoms and associated biochemical abnormalities were thought to be consistent with complicated gallstone disease and specialist opinion was sought [4]. Choledocholithiasis was considered in the differential diagnosis (despite previous cholecystectomy and negative abdominal ultrasound scan). Other diagnoses such as non-alcoholic fatty liver disease, viral and autoimmune hepatitis are usually not associated with severe abdominal pain and were excluded by relevant blood tests. At the suggestion of the specialist gastroenterologist, a magnetic resonance cholangiopancreatography (MRCP) scan (Fig. 1) was requested and carried out urgently.

This confirmed the presence of multiple bile duct calculi following which an ERCP was performed. Endoscopic sphincterotomy was carried out, followed by successful extraction of all stones and common bile duct (CBD) clearance. Following stone extraction, a biliary pigtail stent was inserted to ensure uncomplicated recovery and to reduce the risk of recurrent problems for the remainder of the pregnancy. Prophylactic antibiotics were administered as per guidelines [5].

The patient's symptoms and blood parameters improved rapidly following ERCP and she was discharged home within a few days (Fig. 2-4). She was induced at 38 weeks due to obstetric complications, had a quick vaginal delivery following that, and her postnatal follow-up was uneventful.

\section{DISCUSSION}

Cholelithiasis can occur in pregnancy due to hormone-related changes in cholesterol secretion, causing oversaturation of the bile compared to 


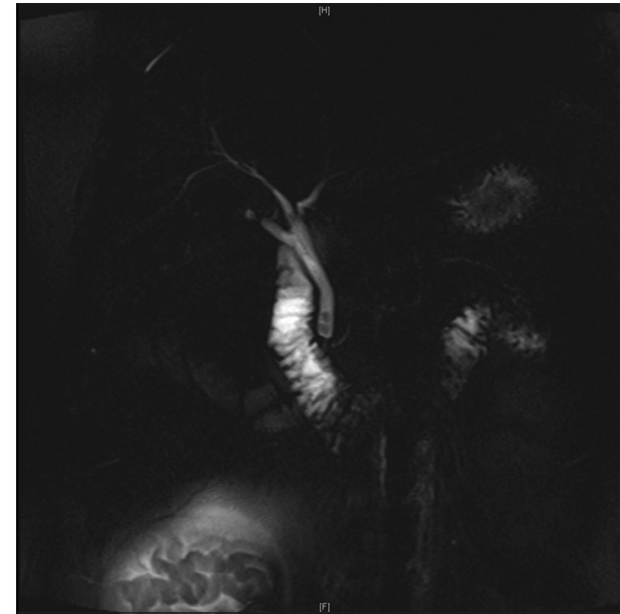

Fig. 1: Magnetic resonance cholangiopancreatography demonstrating the bile duct stones

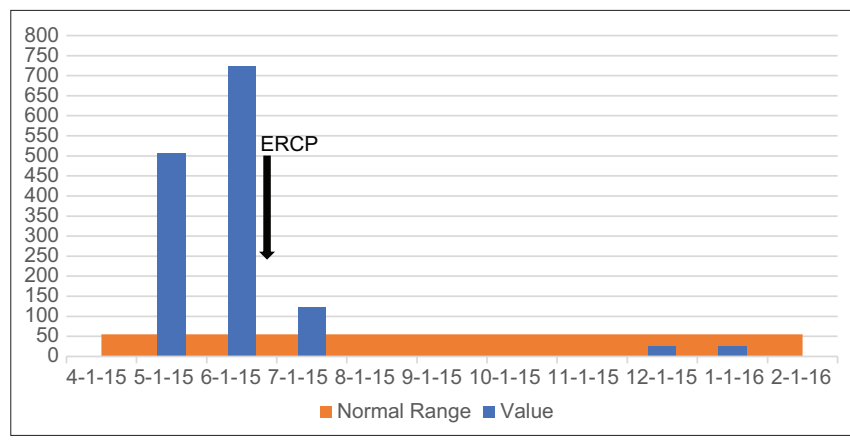

Fig. 2: Value of elevated alanine transferase along with normal ranges before and after magnetic resonance cholangiopancreatography

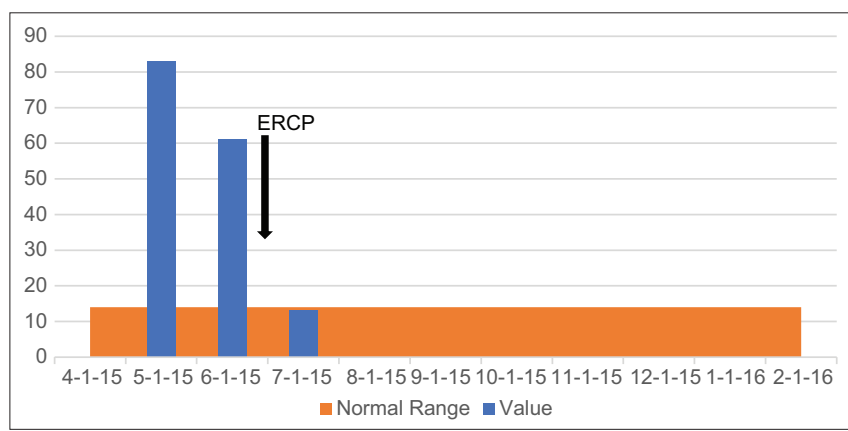

Fig. 3: Value of elevated bile acids along with normal ranges before and after magnetic resonance cholangiopancreatography

bile acids and phospholipids which increases with parity [6]. Up to $10 \%$ of patients develop stones or sludge over the course of each pregnancy, with obesity in particular being a risk factor [2].

Choledocholithiasis complicated by ascending cholangitis is a serious and potentially life-threatening condition in pregnancy, causing preterm labor in up to $10 \%$ of cases [7]. It is, therefore, recommended that ERCP is considered and promptly undertaken to avoid potentially serious complications and adverse outcomes [7]. Elective or semi-elective cholecystectomy after successful ERCP can usually be deferred for after delivery, as it can lead to fetal compromise [8]. Despite concerns regarding the harmful effects of ionizing radiation to the fetus, several retrospective case series have endorsed the safety of this approach during pregnancy (Tang et al., 2009) [9].

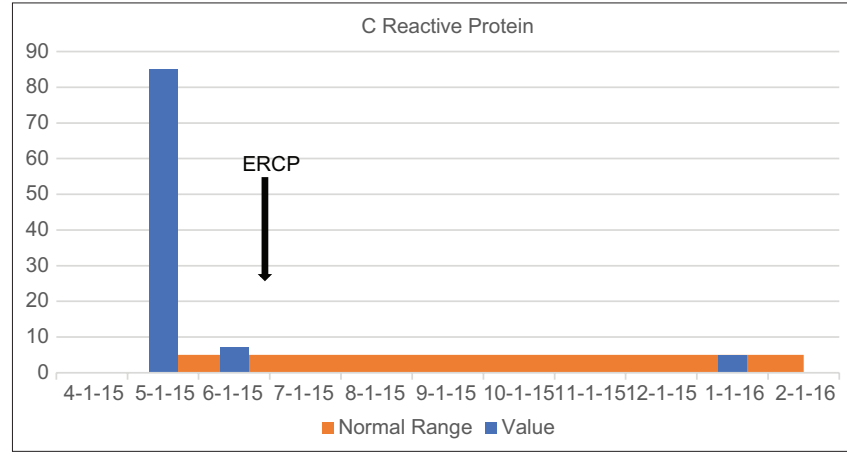

Fig. 4: Value of elevated bile acids along with normal ranges before and after magnetic resonance cholangiopancreatography

The American Society of Gastrointestinal Endoscopy guidance suggests that ERCP is a safe procedure in the setting of symptomatic cholechodolithiasis and its complications during pregnancy [10]. The Society of American Gastrointestinal and Endoscopic Surgeons guidelines recommend either ERCP or laparoscopic cholecystectomy with CBD exploration for choledocholithiasis in pregnancy based on availability and local expertise [7], while European guidelines recommend ERCP during pregnancy for the treatment of choledocholithiasis and its complications (The Italian Association for the Study of the Liver [AISF] 2016) [11].

\section{CONCLUSION}

There is a broad differential diagnosis for obstetric patients presenting with deranged liver tests and epigastric pain and time is of the essence in preventing serious and potentially fatal complications for the mother and fetus. Early involvement of a hepatobiliary specialist and early specific cross-sectional imaging in the form of MRCP is essential for a timely diagnosis and treatment planning.

\section{ACKNOWLEDGMENT}

None to declare.

\section{AUTHORS' CONTRIBUTIONS}

PK, CL, and SM: Write up. RA and HK: Supervision and revision. All authors have contributed to the publication and qualify for authorship.

\section{FINANCIAL DISCLAIMERS/CONFLICTS OF INTEREST STATEMENT}

All authors have no conflicts of interests to declare.

\section{ETHICS APPROVAL}

As this was a case report, formal ethical approval was not required. Permission of the patient has been obtained to publish as case report.

\section{REFERENCES}

1. Maringhini A, Ciambra M, Baccelliere P. Biliary sludge and gallstones in pregnancy: Incidence, risk factors, and natural history. Ann Intern Med 1993;119:116-20.

2. Ko CW, Beresford SA, Schulte SJ, Matsumoto AM, Lee SP. Incidence, natural history, and risk factors for biliary sludge and stones during pregnancy. Hepatology 2005;41:359-65.

3. Fesenmeier M, Coppage KH, Lambers DS, Barton JR, Sibai BM. Acute fatty liver of pregnancy in 3 tertiary care centers. Am J Obstet Gynecol 2005;192:1416-9

4. Linz CM, Murphy CJ, Conteh L, Chen W, Mumtaz K. Acute fatty liver of pregnancy masquerading as choledocholithiasis. Am J Gastroenterol 2016;111:S903.

5. Williams E, Beckingham I, El Sayed G. Updated guideline on the management of common bile duct stones (CBDS). Gut 2017; 66:765-82.

6. Pandey M, Shukla V. Lifestyle, parity, menstrual and reproductive 
factors and risk of gallbladder cancer. Eur J Cancer Prev 2003;12:269-72.

7. The Society of American Gastrointestinal and Endoscopic Surgeons (SAGES). Guidelines for the use of laparoscopy during pregnancy. SAGES Guidel. 2017.

8. Brodsky JB, Cohen EN, Brown BW Jr., Wu ML, Whitcher C Surgery during pregnancy and fetal outcome. Am J Obstet Gynecol 1980;138:1165-7.
9. Tang SJ, Mayo MJ, Rodriguez-Frias E, Armstrong L, Tang L, Sreenarasimhaiah J. Safety and utility of ERCP during pregnancy. Gastrointestinal Endosc 2009;69:453-61.

10. The American Society for Gastrointestinal Endoscopy (ASGE) Guidelines for endoscopy in pregnant and lactating women. Gastrointest Endosc. 2012;76:18-24.

11. The Italian Association for the Study of the Liver (AISF). AISF position paper on liver disease and pregnancy. Dig Liver Dis 2016;48:120-37. 